\title{
Recovering Aluminum from Shredded Aluminum Electrolytic Capacitors by means of a Dry Shaking Table
}

\author{
Hiroshi NAKAZAWA ${ }^{1}$, Yasuo KUDO로 , Shin HASEGAWA ${ }^{2}$ \\ and Kentaro TSUTSUMI ${ }^{2}$ \\ ${ }^{1}$ Graduate School of Engineering, Iwate University, Morioka, Iwate 019-8551, Japan \\ ${ }^{2}$ Faculty of Engineering, Iwate University, Morioka, Iwate 019-8551, Japan
}

\begin{abstract}
Defective aluminum electrolytic capacitors at manufacturing processes are generally shredded to below $3 \mathrm{~mm}$ and buried in landfills. Shredded capacitors contain aluminum, nickel and non-metal materials such as rubber, plastics and fibers. Nickel particles are recovered by magnetic separation, but it is difficult to separate aluminum particle from non-metal materials. We have investigated the recovery of aluminum from shredded capacitors with a laboratory-scale dry shaking table that we developed.
\end{abstract}

Key words: dry shaking table, shredded electrolytic capacitors, aluminum, recovery, non-metal materials

\section{Introduction}

In Japan, defective aluminum electrolytic capacitors at manufacturing processes are shredded, and reclaimed in landfills. They contain aluminum, nickel and non-metal materials such as rubber, plastics and fibers. It is required to recover copper and nickel from the shredded capacitors. Nickel particles are recovered by magnetic separation, but it is difficult to separate aluminum particles from non-metal materials. Typical methods used to separate these materials from each other include manual sorting, eddy current separation and heavy medium separation. The heavy medium separation requires a dry process after the treatment. As defective aluminum electrolytic capacitors are shredded to below $3 \mathrm{~mm}$, they are too small to separate by eddy current separation.

Separation technologies that have been used in the mineral and coal processing are utilized to recover valuables from wastes. A TACUB jig is used to separate three plastics polystyrene (PS), acrylonitrile butadiene styrene (ABS) and polyethylene terephthalate (PET) from each other in a waste treatment plant for copy machines in

Accepted 21 August 2006
Sapporo $^{1)}$. Dense medium separation has been adopted to recover heavy nonferrous metals such as copper, zinc, and lead from automobile shredded residues in waste treatment plants ${ }^{2}$. Shibata, et al. ${ }^{3)}$ reported that four important plastics, namely polyvinyl chloride (PVC), polycarbonates (PC), polyacetal (POM) and poly(phenylene ether) (PPE) were successfully separated from their synthetic mixtures using common wetting agents like sodium ligninsulfonate, tannic acid, Aerosol OT and saponin by flotation.

We attempted the separation of aluminum particles from shredded capacitors using a laboratoryscale dry shaking table. The dry shaking table is shaken in the direction of the slop of the table while typical wet shaking tables in the direction perpendicular to the slop. Separation experiments were carried out by varying several factors such as the tilt of the table, shaking speed, shaking time and so on.

\section{Materials and Methods}

Fig. 1 shows the photograph of the dry shaking table used in this study. The dry shaking table consists of a shaker (Yamato Co. Ltd., SA300), a large stainless vat $(325 \times 280 \times 60 \mathrm{~mm})$ and a 
Recovering Aluminum from Shredded Aluminum Electrolytic Capacitors by means of a Dry Shaking Table

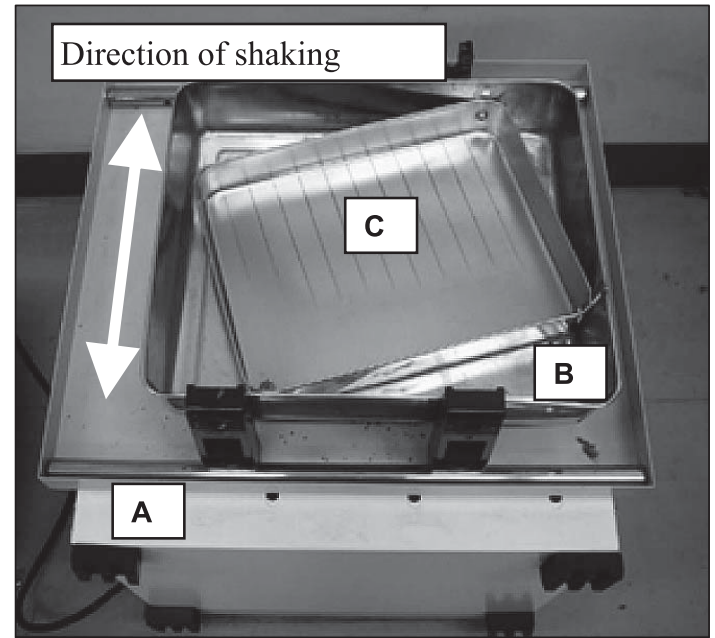

Fig. 1 Dry shaking table.

A: shaker B: large vat C: small vat

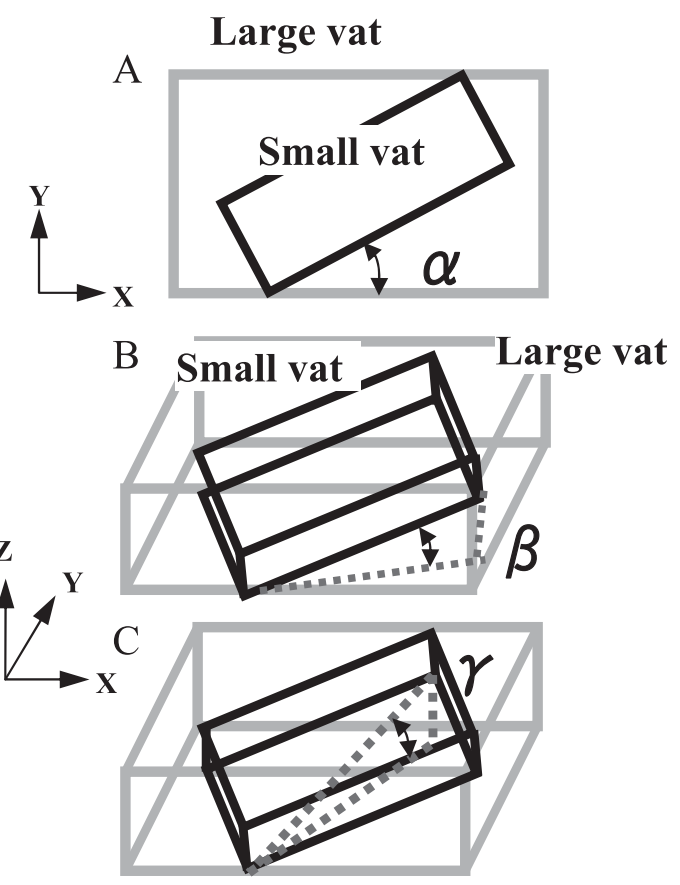

Fig. 2 Tilt of small vat. A: ground plan, B, C: front view

small stainless vat $(240 \times 200 \times 40 \mathrm{~mm})$. The large stainless vat is fixed on the shaker horizontally. The small vat is attached to the large vat with the incline from the horizon with bolts and nuts. The tilt is changeable by adjusting three angles; $\alpha$, $\beta$ and $\gamma$ (Fig. 2). The angle $\beta$ adjusts the longitudinal slope of the small vat whereas the angle $\gamma$ the lateral slop. Both vats reciprocate uniformly in
Table 1 Size distribution of the sample.

\begin{tabular}{lc}
\hline \multicolumn{1}{c}{ size } & weight percent \\
\hline$+2.80 \mathrm{~mm}$ & $0.1 \%$ \\
$-2.80+2.00 \mathrm{~mm}$ & $1.0 \%$ \\
$-2.00+1.18 \mathrm{~mm}$ & $53.2 \%$ \\
$-1.18+1.00 \mathrm{~mm}$ & $13.4 \%$ \\
$-1.00 \mathrm{~mm}$ & $32.3 \%$ \\
\hline
\end{tabular}

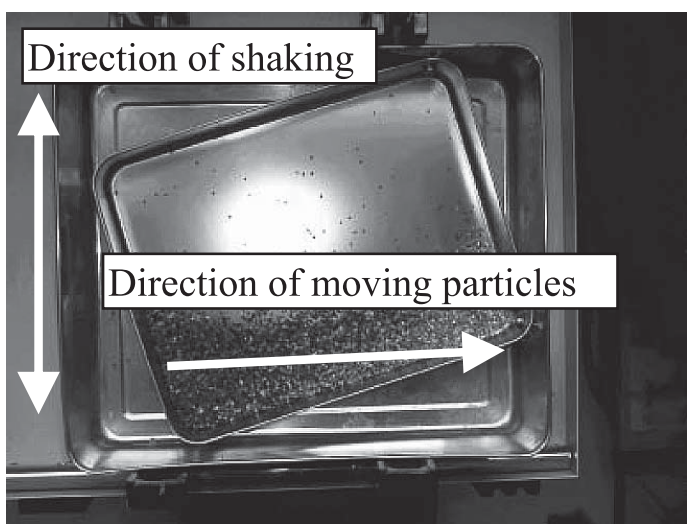

Fig. 3 Directions of shaking and moving particles.

the lateral direction. The shaking speed of the vats is adjustable in the range from 55 to 270 strokes/ min.

The sample used in this study is defective aluminum electrolytic capacitors that were shredded to below $3 \mathrm{~mm}$. It consists of aluminum, nickel, rubber and plastic. The sample was screened after nickel was recovered by dry magnetic separation. Table 1 shows the size distribution of the sample. The size fraction of $-2+1.18 \mathrm{~mm}$ was mainly used for the separation tests. This size fraction was selected because the particles were liberated and because it accounted for $55 \%$ of the sample on a weight basis. The aluminum grade was $52 \%$.

In order to observe the behavior of aluminum particles and non-metal material particles, an aluminum sample and a non-metal material sample were prepared by separating aluminum particles from $\mathrm{a}-2+1.18 \mathrm{~mm}$ fraction sample by handpicking.

Separation experiments were performed in the following procedure. $30 \mathrm{~g}$ of the sample was put at the lower left corner of the small vat. The small vat was shaken for a defined period at the appropriate speed. As the vat was shaken, the particles were moving toward the right end (Fig. 3). After defined time of shaking, sample particles on the small vat were divided into twelve cells with $20 \mathrm{~mm}$ in width and $240 \mathrm{~mm}$ in length. Each cell 


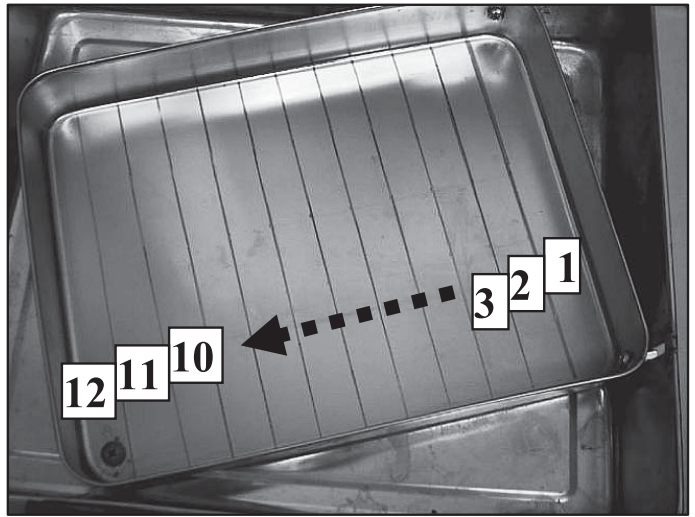

Fig. 4 Number of cells on the small vat.

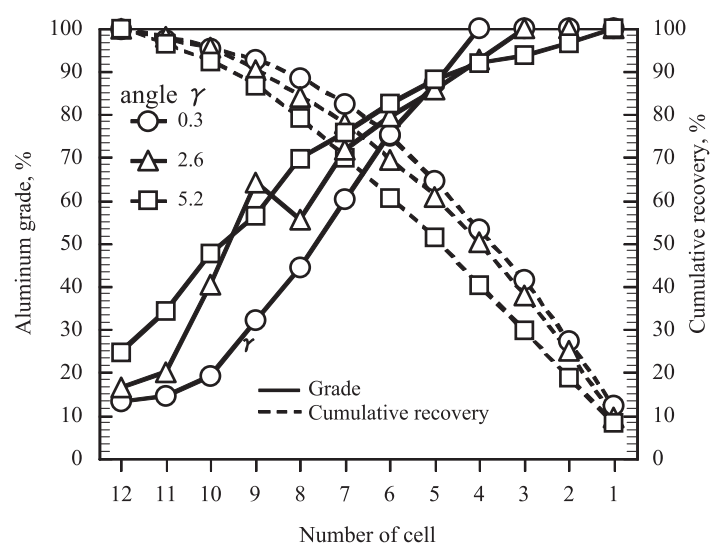

Fig. 5 Effect of angle $\gamma$ on the separation. $\alpha=20^{\circ}, \quad \beta=0^{\circ}$, shaking time:5 min, shaking speed: 270 strokes/min

was numbered 1 to 12 from the right end of the vat as shown in Fig. 4. A product in each cell was collected separately and transformed into $100 \mathrm{ml}$ of a sodium chloride solution with the specific density of 1.4. In the preliminary experiments, we confirmed that only aluminum particles sunk in the sodium chloride solution with that specific density. Sink and float fractions were filtrated separately using a No. 2 filter and rinsed with distilled water several times to remove sodium chloride solution from the particle surfaces. Sinks and floats were dried at $60^{\circ} \mathrm{C}$ and weighed. Recovery rate and grade of aluminum were calculated

\section{Results and Discussion}

In preliminary experiments, we optimized the tilt of the small vat by adjusting three angles; $\alpha, \beta$ and $\gamma$. Fig. 5 represents one of results of those experiments. The value of $\gamma$ was varied from $0.3^{\circ}$ to

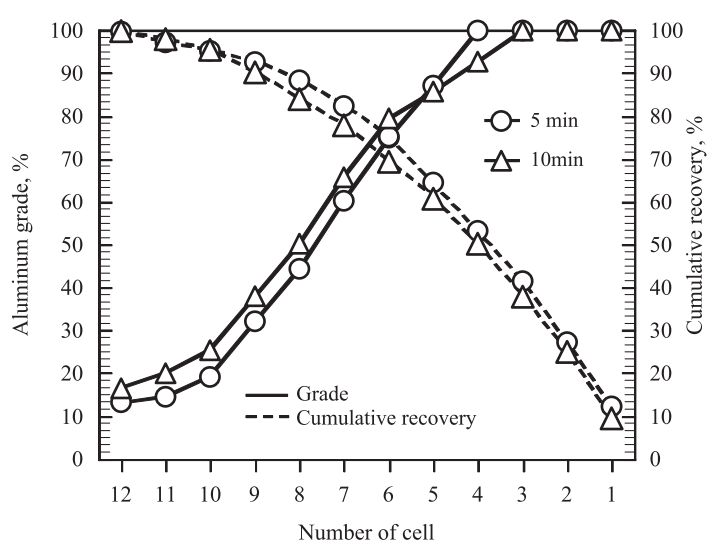

Fig. 6 Effect of shaking time on the separation sample:30 g, shaking speed: 270 strokes/min

$5.2^{\circ}$ while $\alpha$ and $\beta$ were fixed at $20^{\circ}$ and $0^{\circ}$, respectively. The more the angle $\gamma$ increases, the higher the right end of the vat becomes. The shaking speed was 270 strokes/min, and shaking time was $5 \mathrm{~min}$. Aluminum grade of the products obtained in the cells from No. 1 to No. 4 was almost $100 \%$ at $\gamma$ value of $0.3^{\circ}$. For $\gamma=2.6^{\circ}$, aluminum grade of the product in No. 4 cell was $92 \%$. For $\gamma=5.2^{\circ}$, the aluminum grade of products in No. 2 cell and No. 3 cell was decreased to $96 \%, 92 \%$ respectively, and the product in No. 1 cell only had pure aluminum. The cumulative recovery of aluminum obtained from No. 1 cell to No. 4 cell decreased with increase in $\gamma$. It could be because the amount of aluminum particles conveyed toward the higher right side of the vat decreased with increase in $\gamma$ value. Therefore, following separation experiments were performed at $\alpha=20^{\circ}, \beta=0^{\circ}$ and $\gamma=0.3^{\circ}$.

In order to evaluate the effect of shaking speed on the separation, the separation experiments were carried out by varying shaking speed from 55 to 270 strokes/min (maximum speed). It was found by visual observation that the separation was difficult at the shaking speed below 190 strokes/min. Good separation results were obtained at 270 strokes/min. This shaking speed was chosen for the further experiments.

The effect of shaking time on the separation was examined by shaking the vat for $5 \mathrm{~min}$ or 10 min. The results obtained are shown in Fig. 6. The aluminum grade of products obtained in each cell from No. 1 to No. 4 was almost $100 \%$ after shaking time of $5 \mathrm{~min}$. The cumulative recovery from No. 1 cell to No. 4 cell after 10 min shaking was higher than that after 5 min shaking. The aluminum grade of the product in No. 4 cell was $92 \%$ 
after shaking time of 10 min while almost $100 \%$ for 5 min shaking, indicating that non-metal material particles tended to move toward the right side of the vat with increase in shaking time. Hereafter experiments were conducted at shaking time of 5 min.

Fig. 7 shows the effect of the amount of sample on the separation. The experiments were performed using the samples of $30 \mathrm{~g}$ and $60 \mathrm{~g}$. For the former amount, the aluminum grade of the products obtained in each cell from No. 1 to No. 4 was almost $100 \%$, and $53 \%$ of aluminum was recovered in those four cells. For the latter amount, the grade of the products in No. 1 cell and No. 2 cell was almost $100 \%$, but the grade of the product in No. 3 cell decreased to $87 \%$. The amount of aluminum obtained in No. 1 cell and No. 2 cell accounted for $17 \%$ of aluminum of the sample. It was found that the optimum amount of sample for this test is $30 \mathrm{~g}$.

To improve the cumulative recovery of aluminum, separation experiments were performed four times successively. The experiment procedure was as followed: After a separation experiment conducted by the procedure described above (named Run $1^{\text {st }}$ ), high grade (over about 90\%) part

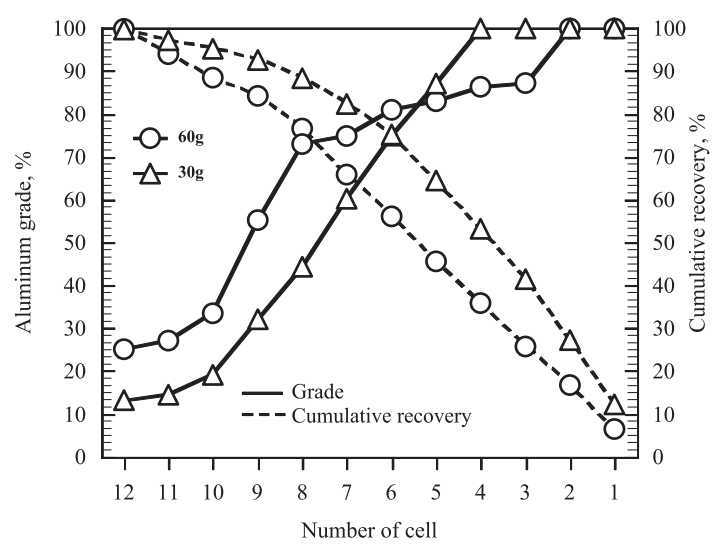

Fig. 7 Effect of amount of sample on the separation shaking time: $5 \mathrm{~min}$, shaking speed: 270 strokes/ $\min$

Table 2 Results of consecutive separation experiments. shaking time: $5 \mathrm{~min}$, shaking speed: 270 strokes $/ \mathrm{min}$

\begin{tabular}{llc}
\hline No. & Concentrate & $\begin{array}{c}\text { Cumulative } \\
\text { Recovery }\end{array}$ \\
\hline Run 1st & No. 1-4 & $52 \%$ \\
Run 2nd & No. 1-3 & $70 \%$ \\
Run 3rd & No. 1-2 & $79 \%$ \\
Run 4th & No. 1 & $82 \%$ \\
\hline
\end{tabular}

of products was collected on a basis of cell unit followed by conducting separation experiments using the remainder of the sample. These experiments were named Run $2^{\text {nd }}$, Run $3^{\text {rd }}$ and Run $4^{\text {th }}$, respectively. The results obtained are presented in Table 2. For Run $1^{\text {st }}$, the products in the cell from No. 1 to No. 4 were collected while the products in No. $1-$ No. 3 cells for Run $2^{\text {nd }}$, in No. 1-No. 2 cells for Run $3^{\text {rd }}$, and in No. 1 cell for Run $4^{\text {th }}$ were collected as concentrates. After the $4^{\text {th }}$ separation experiment, the cumulative recovery of aluminum amounted to $82 \%$ with the aluminum grade of $95 \%$.

To examine the moving behavior of aluminum particles and non-metal material particles, experiments was conducted using samples of aluminum or non-metal material particles. Fig. 8 shows the weight percentage of products obtained in each cell after shaking time of 5 min using $15 \mathrm{~g}$ of an aluminum sample or a non-metal material sample. The distribution trend of non-metal material particles as a function of each cell was almost the same as that of aluminum particles. Fig. 9 shows the weight percentage of products obtained in each cell after shaking time of 5 min using the mixture of $15 \mathrm{~g}$ aluminum sample and $15 \mathrm{~g}$ non-metal material sample. It was observed that aluminum particles were conveyed more quickly toward the right end than non-metal material particles in the case the separation test was carried out using the mixture. Non-metal material particles did not exist in the right side of the vat (No. 1-No. 5 cells). Quick occupation of aluminum particles in the part of the right side could prevent non-metal ma-

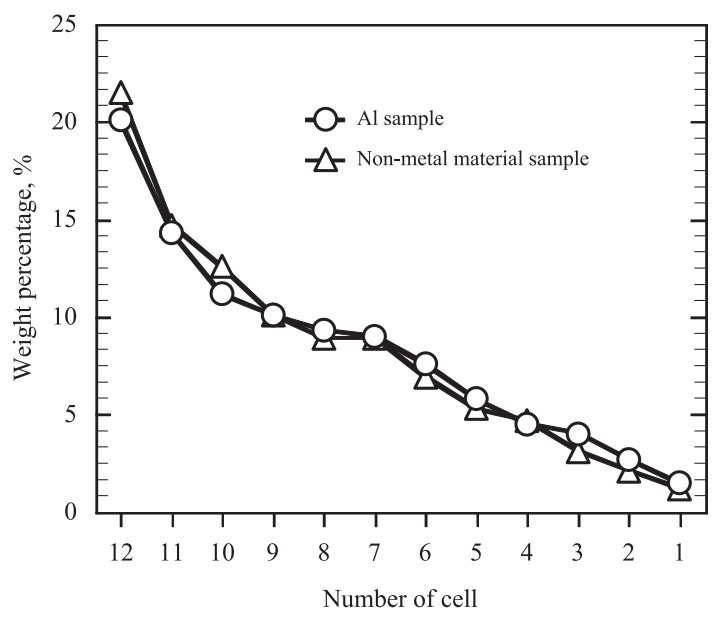

Fig. 8 Distribution of aluminum and non-metal material as a function of cell using each sample shaking time:5 min, shaking speed: 270 strokes $/ \mathrm{min}$ 


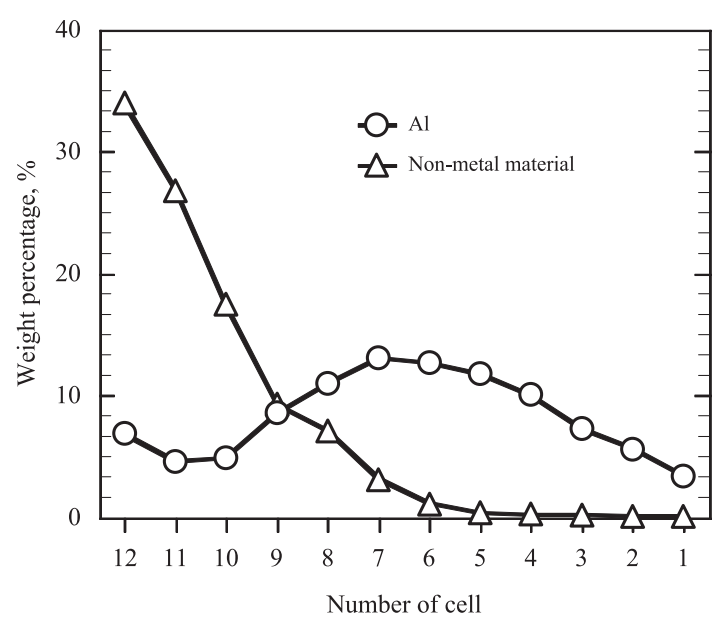

Fig. 9 Distribution of aluminum and non-metal material as a function of cell using the mixture of aluminum and non-metal material shaking time: $5 \mathrm{~min}$, shaking speed: 270 strokes/min

terial particles from moving toward the right side.

Several researchers have investigated the separation of plastics from each other using an air table. $\mathrm{Oi}^{4}$ investigate the separation of polyethylene (PE) and polyvinyl chloride (PVC) from each other with an air table. Dodbiba, et al. ${ }^{5}$ have performed separation experiments using 50/50\% artificial mixtures of PVC and polypropylene (PP) (size friction of $-3.36+2.38 \mathrm{~mm}$ ). They indicated that products of PVC and PP with a grade of 99.90 and $95.84 \%$ respectively have been achieved with recoveries higher than $96.70 \%$ at the optimum operating condition. In the case of air tables, a porous deck can be tilted from inlet end to discharge end and from side to side. The porous deck vibrates eccentrically the longitudinal direction. Low-density particles float on the deck with the upward airflow blown through below the porous deck, but high density particles settle on the deck, resulting in the longitudinal movement of high density particles. High density particles are col- lected at the longitudinal end.

In the case of our shaking table, high density particles (aluminum particles) were also conveyed towards the right side of the vat. The vat of our shaking table, however, is shaken in the direction of the small side of the vat (in lateral direction) while the deck of an air table in the longitudinal direction. The mechanism of separation with the dry shaking table is not clear, but it could be different from that of air tables. Further investigation is necessary to elucidate the mechanism of the separation.

\section{Conclusions}

To recover aluminum particle from shredded aluminum electrolytic capacitors, which contained rubber, plastics and fibers besides aluminum, we have developed the dry shaking table and carried out separation experiments. Aluminum particles moved preferentially, and the grade of the concentrate was almost $100 \%$ with the recovery rate of $53 \%$ at the optimum operating condition.

\section{References}

1. M. Tsunekawa, S. Ogawa, B. Naoi, T. Hirajima, N.Hiroyoshi: Proc. 7th Int. Symp. on East Asian Resources Recycling Technologies, Tainan, National Cheng Kung University, pp. 155-158 (2003)

2. S. Mori, M. Nonaka, R. Matsufuji, T. Fujita, M. Futamata, M. Hata: Shigent-to-Sozai, 109, pp. 811-817 (1993)

3. J. Shibata, S. Matsumoto, H. Yamamoto, E. Kusaka, Pradip: Int. J. Min. Pro., 48, 3-4, pp. 127-134 (1996)

4. E. Oi: Shigen to Kankyou, 9, 2, pp. 15-20 (2000)

5. G. Dodbiba, A. Shibayama, T. Miyazaki, T. Fujita: Physical Separation in Science and Engineering, 12, 2, pp. 71-86 (2003) 